\title{
BREVE NOTA SOBRE LA IDEA DE «PATERNALISMO» EN LA OBRA DE ERNESTO GARZÓN VALDÉS
}

\author{
Macario Alemany \\ Universidad de Alicante
}

RESUMEN. El autor hace un balance de las tesis principales de Ernesto Garzón Valdés sobre el concepto y la justificación del paternalismo jurídico, destacando su relevancia en la iusfilosofía en lengua española y poniéndolas en relación con el conjunto de su obra.

Palabras clave: Ernesto Garzón Valdés, paternalismo, perfeccionismo, autonomía.

ABSTRACT. The author takes stock of the main theses about the concept and justification of legal paternalism chez Ernesto Garzón Valdés, emphasizing their relevance in the Spanish legal philosophy and connecting them with his overall work.

Keywords: Ernesto Garzón Valdés, paternalism, perfeccionism, autonomy. 


\title{
1. RELEVANCIA DE LA APORTACIÓN DE ERNESTO GARZÓN VALDÉS A LA DISCUSIÓN SOBRE EL PATERNALISMO
}

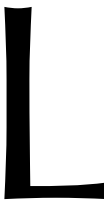

\begin{abstract}
a relevancia de la doctrina de GARZÓN VALDÉS sobre el paternalismo puede contemplarse «ad extra», es decir, el impacto del artículo en la comunidad iusfilosófica y «ad intra», es decir, el papel que la idea de paternalismo juega en la construcción de otras tesis del autor.
\end{abstract}

«Ad extra» no es exagerado decir que con este artículo GARZÓN VALDÉS introduce la discusión sobre el paternalismo jurídico en la iusfilosofía en lengua española ${ }^{1}$.

Si bien la idea de paternalismo, la analogía entre el poder paternal y el poder político, ya está presente en la filosofía griega y reaparece, variando su modulación y alcance, recurrentemente en relación con el tema de la justificación del poder estatal, sería en los años setenta del pasado siglo, y a partir de una serie de influyentes aportaciones de Gerald DwORKIN ${ }^{2}$, Joel FEINBERG ${ }^{3}$ y John RAWLS ${ }^{4}$, cuando se genera una intensa discusión sobre la definición correcta de paternalismo jurídico y la plausibilidad de interferencias paternalistas justificadas. El artículo de GARZÓN VALDÉS, «¿Es éticamente justificable el paternalismo jurídico?» ${ }^{5}$, enlaza directamente con los presupuestos y tesis de estos autores y, como decía, fue el punto de partida de la discusión en el ámbito latinoamericano.

«Ad intra» la concepción del paternalismo de GARZÓN VALDÉS es muy importante en la construcción de su filosofía jurídica, moral y política, puesto que el paternalismo es clave en la defensa de uno de los propósito fundamentales que han guiado toda su obra, esto es, «la justificación del Estado social de Derecho desde un punto de vista liberal» ${ }^{6}$. Recordemos que, a juicio de GARZÓN VALDÉs, en contra de la fundamentación liberal del Estado social suele aducirse «el peligro del paternalismo (que sería el primer paso hacia la dictadura) y la diferencia radical que existiría entre deberes positivos y negativos» ${ }^{7}$. De ahí que nuestro autor haya mostrado un gran interés en sostener dos tesis: primera, que hay casos de paternalismo justificado y, segunda, cuestionar la asimetría moral entre acciones y omisiones. En defensa de ambas tesis GARZÓN VALDÉS ha argüido que la propia aceptación del principio de daño (incuestionable desde el

${ }^{1}$ Véase: GARZÓN VALDÉs, 1988: «¿Es éticamente justificable el paternalismo jurídico?», en DOXA, 5, pp. 155-173. Una primera versión de este trabajo fue publicada en Revista Latinoamericana de Filosofía, XIII (1987), n. ${ }^{\circ}$ 3. Ha sido reeditado en GARZÓN VALDÉs, 1993: Derecho, ética y política, Madrid: CEC, pp. 361-378; y, en versión inglesa, con el título: «On Justifying Legal Paternalism», en Ratio Iuris, 3 (1990), pp. 173-184. He realizado un estudio detallado sobre la idea de paternalismo en la obra de Ernesto GARZÓN en ALEMANY, 2006: El paternalismo jurídico, Madrid: Iustel, cap. 3.

${ }_{2}$ DwORKIN: «Paternalism», en WASSERSTROM (ed.), 1971: Morality and the Law, Belmont: Wadsworth Publishing Co., pp. 107-126. Existen las siguientes reediciones del mismo: en The Monist, 56, n. ${ }^{\circ} 1$ (1972), pp. 6484; en SARTORIUS, 1987: Paternalism, Minneapolis: University of Minnesota Press, pp. 19-34; y en FeINBERG, y Coleman, 2000: Philosophy of Law, Belmont: Wadsworth, 2000, pp. 271-280. Existe una traducción del mismo al castellano realizada por Jorge M. SEÑA e incluida en la colección de ensayos: Derecho y Moral. Ensayos analíticos, editada por Jerónimo BETEGÓN y Juan Ramón DE PÁRAMO, publicada en 1990.

${ }^{3}$ FeINBERG: «Legal Paternalism», en Canadian Journal of Philosophy, 1, n. ${ }^{\circ}$ 1, pp. 106-124.

${ }^{4}$ Rawls, 1997: Teoría de la Justicia, México: Fondo de Cultura Económica, pp. 230 y ss.

5 GARZÓN VALDÉS, 1988.

${ }^{6}$ GaRZÓn ValdÉs, 1993: Derecho, ética y política, Madrid: CEC, p. 12.

7 GARZÓN VALDÉS, 1993. 
punto de vista liberal) conduce a la aceptación de un paternalismo restringido y de la práctica de medidas asistenciales que impongan sacrificios mínimos.

\section{LA POSICIÓN DE GARZÓN VALDÉS SOBRE EL CONCEPTO DE PATERNALISMO}

El punto de partida de GARZÓN VALDÉS es indagar la plausibilidad moral de un principio jurídico-paternalista que se enuncia de la siguiente manera: «Siempre hay una buena razón a favor de una prohibición o de un mandato jurídico, impuesto también en contra de la voluntad del destinatario de esta prohibición o mandato, cuando ello es necesario para evitar un daño (físico, psíquico o económico) de la persona a quien se impone esa medida» ${ }^{8}$.

El contenido de este principio sería el concepto de paternalismo jurídico de GARZÓN VALDÉS, en el que podemos distinguir dos elementos:

1) La forma de la acción paternalista: el establecimiento de una prohibición u obligación jurídica.

2) La finalidad: evitar daños físicos, psíquicos y/o económicos al destinatario mismo de la prohibición u obligación.

Así, por ejemplo, constituiría un caso de paternalismo jurídico la obligación de llevar cinturón de seguridad en los coches o el casco en las motos, la prohibición del consumo de drogas peligrosas, etc.

Sobre esta conceptualización del paternalismo me parece interesante hacer ahora dos consideraciones:

1) El primer elemento de la definición hace que ésta sea inadecuadamente restringida: con frecuencia la persona tratada paternalistamente no es la destinataria de la norma de mandato que consideramos paternalista (piensen en la prohibición que pesa sobre los farmacéuticos de expedir medicamentos sin receta, o la prohibición del tráfico de drogas orientada a evitar daños en el consumidor, etc.). Otras veces el paternalismo consiste en un permiso dirigido al agente paternalista, por ejemplo un médico, para actuar de cierta manera, por ejemplo imponiendo un tratamiento, de manera que el sujeto tratado paternalistamente, el paciente en este caso, no puede ejercer con éxito una acción judicial contra el médico. Finalmente, en ocasiones el paternalismo jurídico ni siquiera se concreta en normas regulativas sino que se ejerce a través de normas de competencia que dejan a los sujetos tratados paternalistamente en una situación de incompetencia (frente a lo que se considera un nivel normal o pleno de competencia) o de sujeción frente a ciertas autoridades o particulares.

Todo ello apunta a que el paternalismo jurídico sería mejor definido como el ejercicio de un poder jurídico, el cual puede tener como resultado poner al sujeto tratado paternalistamente en una situación de deber, no derecho, sujeción y/o incompetencia'.

\footnotetext{
8 GARZÓN VALDÉS, 1988: 156.

9 Sobre esta propuesta conceptual, véase: Alemany, 2006: cap. V; y Alemany, 2005: «El concepto y la fundamentación del paternalismo jurídico», en Doxa, 5, pp. 265-306.
} 
2) La segunda consideración se refiere a la finalidad propia del paternalismo. GARZÓN VALDÉS circunscribe el paternalismo — creo que muy acertadamente- a la finalidad de evitar daños de tipo físico, psíquico y/o económico. Esto le permite distinguir al paternalismo de otras figuras afines y, muy particularmente, del moralismo jurídico, cuya finalidad sería evitar daños morales a los individuos, y del perfeccionismo que tiene un carácter maximizador y moralizante: perfeccionar el carácter de los sujetos.

\section{LA POSICIÓN DE GARZÓN VALDÉS SOBRE LA JUSTIFICACIÓN DEL PATERNALISMO}

GARZÓN VALDÉS considera que el paternalismo jurídico estaría justificado si se cumplen las siguientes condiciones necesarias y conjuntamente suficientes: 1) se constata fehacientemente una incapacidad básica del sujeto tratado paternalistamente y 2) la medida se orienta a la superación del déficit creado por la incompetencia ${ }^{10}$.

Estoy de acuerdo con que éstas sean condiciones necesarias de la justificación del paternalismo, pero no con que sean suficientes: en primer lugar, creo que no basta con que la medida se oriente sinceramente a superar el déficit generado por la incompetencia sino que, además, debe ser necesaria y adecuada (es decir, proporcionada) y, junto a esto, debe ser racional presumir que el sujeto tratado paternalistamente aceptaría tanto la posibilidad general de ser tratado así como la concreta interferencia paternalista.

Que sea racional aceptar la posibilidad general de ser tratado paternalistamente depende, a mi juicio, de que haya los suficientes controles y contrapesos, propios de un Estado constitucional, como para desvirtuar el contra-argumento de la pendiente resbaladiza: de la misma manera que un individuo difícilmente aceptaría ser internado en una institución psiquiátrica que mostrara una total opacidad sobre su funcionamiento interno o aislamiento absoluto con el exterior, parece difícil aceptar racionalmente el paternalismo de una administración sin controles y limitaciones.

Finalmente, que sea racional aceptar una concreta medida paternalista depende de los fines y valores del propio individuo, de ahí que, en ocasiones, presunciones generales sobre lo que constituye un daño para los sujetos deban ceder en un caso concreto ante la evidencia de la existencia de ciertos propósitos individuales: éste sería, por ejemplo, el caso de algunas huelgas de hambre políticas en las que se ha alimentado forzosamente a los huelguistas sobre el presupuesto general de que eso es por su propio bien, pero sin respetar sus particulares fines y valores ${ }^{11}$.

\footnotetext{
10 GARZÓn VALDÉs, 1988: 167.

11 Sobre esta propuesta de justificación, véase igualmente: Alemany, 2006: cap. V; y Alemany, 2005.
} 